\title{
Verarbeitung und qualitative Zusammensetzung des Zirkons.
}

(Mit, 2 Holzschnitten.)

Von Ed. Linnemann.

(Aus dem chemischen Laboratorium der k. k. deutschen Universität zu Prag.)

(Vorgelegt in der Sitzung am 7. Mai 1885.)

Eine praktische Frage, deren Erledigung jedoch zugleich auch ein rein wissenschaftliches Interesse bot, veranlasste mich, grössere Mengen von Zirkon auf Zirkonerde zu verarbeiten. Die Beobachtungen, welche ich hierbei zu machen Gelegenheit hatte, scheinen mir der Mittheilung werth und sind in dem Nachfolgenden enthalten.

Der Zirkon gehört zu den härtesten und am schwersten aufschliessbaren Silicaten. Um das Pulverisiren des Zirkons, welcher behufs Aufschliessung in ein sehr zartes Pulver verwandelt werden muss, zu erleichtern, pflegte man die Zirkonkrystalle rothglühend in kaltes Wasser zu werfen, wodurch sie in Folge vieler entstehender Risse matt werden und sich auch etwas besser zerkleinern lassen.

Dieser Zweck kann bei weitem viel vollständiger erreicht werden, wenn man Zirkonkrystalle der Einwirkung der Dämpfe von Fluorwasserstoffsäure aussetzt. Sie zerfallen hiebei zu einem gröblichen Pulver, welches sich in einem Eisenmörser sehr leicht pulverisiren lässt. Es verhielten sich in dieser Beziehung Zirkonkrystalle vom Ural und von Nordearolina ganz gleich. Allerdings zerfallen nicht alle Krystalle in gleichem Maße. Manche fast vollständig, bei anderen erhalten sich die besser ausgebildeten Endfächen im Zusammenhange, zerfallen aber trotzdem unter dem Drucke des Pistills leicht zu Pulver.

Die Zirkonkrystalle genannten Ursprungs sind demnach mit unzähligen feinen Sprïngen versehen, welche von einem Silicate 
ansgefüllt sind, das durch gasförmige Flusssäure leicht und vollständig aufgeschlossen wird, während die Krystallgrundmasse des Zirkons unangegriffen bleibt.

Man hat so zugleich ein Mittel an der Hand, die krystallinische Grundmasse der Zirkonkrystalle in vollkommen reinem Zustande zur Verarbeitung zu bringen.

Wie bei diesem Präpariren des Zirkons behufs Aufschliessung vorgegangen wurde, mag ans folgenden beispielsweisen Angaben ersichtlich sein.

$193 \mathrm{Grm}$. ganze Zirkonkrystalle aus Nordcarolina wurden unter häufigem Wenden zehn Tage lang in einer Platinschale den Dämpfen von Flusssäure ausgesetzt and die zerfallene, zerbröckelte Masse mit Wasser befeuchtet, abermals bis zur Sättigung mit Flusssäuregas behandelt. Das Ganze wurde alsdann unter Zusatz von Wasser längere Zeit auf dem Wasserbade erwärmt und vollständig, zuletzt unter Zusatz von Salzsäure ausgewaschen. Die erhaltene Lösung enthält die Bestandtheile des zersetzbaren Silicates. Das ausgewaschene Zirkonpulver, dessen Gewicht nach scharfem Trocknen $187 \mathrm{Grm}$. betrug, liess sich in einem Eisenmörser leicht zu einem so feinen Pulver zerreiben, dass es durch das feinste Seidensieb ging. Durch Behandeln mit etwas Königswasser wurde das aus dem Mörser stammende Eisen entfernt, worauf das so präparirte Zirkon nach dem Waschen und scharfen Trocknen ein schneeweisses, schweres, der Pfeifenerde gleichendes Pulver darstellte, dem weder Flusssäure noch Königswasser weitere merkbare Mengen an Mineralsubstanz entzogen. Das Gewicht betrug $180 \mathrm{Grm}$., circa $93 \%$ der ursprünglichen Zirkonkrystalle.

Demnach enthielten die erwähnten Krystalle Nordcarolinazirkon etwa $4 \%$ eines durch Flusssäure aufschliessbaren Silicates, dem auf Grund der vorgenommenen qualitativen Untersuchung: obiger Lösung dienachfolgenden metallischen Elemente za Grunde liegen:

$$
\mathrm{Na}, \mathrm{Ka}, \mathrm{Li}, \mathrm{Mg} \text {, Ca, Al. Fe, Zr. }
$$

Uralzirkon, grosse, einzelne, aber schlecht ausgebildete, dem Granattypus sich nähernde Krystalle, gaben bei gleicher Behandlung folgendes Resultat. Sie enthielten circa $5 \%$ durch 
Flusssäure aufschliessbares Silicat, das mit Ausnahme des Li dieselben metallischen Elemente enthielt, wie der Nordcarolinazirkon. Man erhielt etwa $94 \%$ des feingepulverten, zum Aufschliessen fertigen Zirkons.

Das Aufschliessen des Zirkons ist nach den bis jetzt empfohlenen Methoden eine schwierige Sache und im Grossen unmöglich. Von den vorgeschlagenen Flussmitteln: Ätznatron, kohlensaures Natron, saures schwefelsaures Natron und Fluornatrium greift das Letztere wohl das Zirkon am leichtesten an, allein die Methode ist aus anderen Grüden im Grossen gleichfalls nicht anwendbar. Die anderen Flussmittel greifen das Zirkon zu langsam und unvollständig an, man muss Stunden lang bei den höchsten erreichbaren Temperaturen erhitzen, die aufgeschlossene Menge Zirkon ist immer nur gering, die Defecte an den Tiegeln so gross, dass, Alles in Allem genommen, diese Methoden im Grossen nicht anwendbar sind.

Auf Grund zahlreicher, zeitraubender Versuche ist es mir gelungen, eine Methode aufzufinden, welche gestattet, $25 \mathrm{Grm}$. Zirkon in 15 Minuten mit einem einfachen Bunsenbrenner aufzuschliessen, eine Methode, welche sich in anderen Fällen zum Aufschliessen von Silicaten glänzend bewährt hat und für das Laboratorium eine angenehme Acquisition werden wird.

Das Flussmittel besteht in einer Mischung von Ätznatron und wenig Fluornatrium. Eine derartige Mischung greift hinreichend fein gepulverten Zirkon noch rascher und leichter an als Fluornatrium allein. Indem anfangs Kieselfluornatrium und Floormetalle entstehen, werden diese durch das schmelzende $\mathrm{Na}(\mathrm{OH})$ in kieselsaures Natron und Metalloxyde umgewandelt, wobei unter Aufschäumen der Schmelze Wasserdampf frei wird und das Fluornatrium immer wieder regenerirt wird. Desshalb reicht auch eine nur kleine Menge Fluornatrium vollkommen ans. Mit dem Aufhören der Wasserbildung, d. h. dem Aufschäumen der Schmelze, ist die Reaction zu Ende und das Silicat aufgeschlossen.

Das Aufschliessen des Zirkons wurde in einem circa $200 \mathrm{Ce}$. fassenden, mit dem Deckel $250 \mathrm{Grm}$. schweren Silbertiegel von $75 \mathrm{Mm}$. oberem Diameter und $70 \mathrm{Mm}$. Höhe von beiläufig $2 \mathrm{Mm}$. Wandstärke vorgenommen. 
Man gibt 100 Grm. zuvor bis zum ruhigen Fliessen ge-. schmolzenen Ätznatrons, $10 \mathrm{Grm}$. reines Fluornatrium ${ }^{1}$ and 25 Grm. präparirten, durch ein feines Seidensieb passirten Zirkons in den Tiegel und erwärmt mit einem guten Bunsenbrenner. Bei vollem Gasdruck beginnt das Aufschäumen rasch und bei Aufmerksamkeit, zeitweiligem Entfernen des Brenners und Regulieren desselben, lässt sich ein Überschäumen leicht vermeiden. Nach etwa 10-15 Minuten ist die Hauptreaction vorüber und aus der ruhig fliessenden Masse entweichen ohne Aufschäumen gleichmässig Gasblasen. Diese nachfolgende Reaction ribut daher, dass die etwas weniger feinen Theileben des Zirkonpulvers längerer Zeit bedürfen, um von der Schmelze gelöst zu werden. Um dies für alle Fälle möglichst vollständig zu erreichen, erhitzt man mit bedecktem Tiegel etwa noch eine halbe Stunde, wobei Dunkelrothgluth erreicht wird und man zweckmässig einige Male mit einem dicken Platindraht umrührt. Auch nach Verlauf dieser Zeit ist die Masse noch dünnflüssig. Man giesst sie in einer Siberschale un dümen Knehen aus, welche sich beim Erkalten leicht loslösen and sich in heissem Wasser leicht unter Zurïcklassung von Zirkonerdenatron und die, die Zirkonerde begleitenden Oxyde zertheilt, während Natron, kieselsaures Natron und Fluornatrium in Lösung gehen. In dieser kalischen Lösung konnte von Oxyden, die aus dem Zirkon stammen, nur Thonerde und Lithion nachgewiesen werden.

Der unaufgeschlossene Zirkon bleibt neben etwas KieseIsäure beim späteren Lösen des rohen Zirkonerdenatrons in Salzsäure zurück und betrug nach Entfernung der Kieselsäure mittelst Natronlauge im Mittel mehrerer Versuche im Maximum 1.5\% des in Arbeit genommenen präparirten Zirkons.

Der in Wasser unlösliche Theil der Schmelze, das rohe Zirkonerdenatron, wird möglichst vollständig durch Decantation gewaschen, wobei man aufbören muss, sobald das Absetzen

1 Das Fluornatrium stellt man aus käuflicher reinster Flusssänre dar, indem man dasselbe mit reinem kohlensauren Natron neutralisirt und so lange versetzt, bis kein Niederschlag mehr entsteht. Die Lösung wird pach dem Filtrìen mit Salzsäure schwach angesäuert, mit Schwefelwasserstoff ausgefäilt, filtrirt, in Platingefässen zur Trockne gebracht, und scharf getrocknet. 
nicht mehr gut erfolgt, was stets eintritt, sobald bei zunehmendem Auslaugen der Salzgebalt des Waschwassers stark abgenommen hat. Man sammelt auf Filtern, lässt lufttrocken werden und trocknet auf dem Wasserbade.

25 Grm. präparirten Zirkons geben auf diese Weise im Durchschnitte von sieben verschiedenen Versuchen 26 Grm. rohes Zirkonerdenatron, wobei die Ausbente in den extremsten Fällen zwischen 23.5 und $30 \mathrm{Grm}$. schwankte.

Zur weiteren Verarbeitung des "rohen Zirkonerdenatrons" wird dasselbe mit überschüssiger verdünnter Salzsäure gelöst, wiederholt zur Trockne gebracht und wie zur Abscheidung. derKieselsäure behandelt. Es scheidet sich nicht nur Kieselsäure ab, sondern es entweicht auch Flusssäure und eine sehr kleine Menge unaufgeschlossenen Zirkons wird entfernt.

Die Trennung des Zirkonchlorids von allen anderen aus dem Zirkone stammenden Metallchloriden geschieht in der einfachsten Weise durch Behandeln mit einer Mischung von ranchender Salzsäure spec. Gew. $=1 \cdot 17$, absolutem Alkohol und Äther, in welcher Mischung das $\mathrm{ZrCl}_{4}$ fast unlöslich ist, sämmtliche anderen Chloride bis auf ganz geringe Mengen löslich sind.

Das unreine, vor allem Natriumchlorid haltende $\mathrm{ZrCl}_{4}$ wird zll diesem Zwecke zunächst auf dem Wasserbade vollständig getroeknet, auf das feinste gepulvert und durch ein feines Sieb getrieben. Das stanbfeine Pulver wird nun in einer geräumigen Flasche mit soviel reiner Salzsäure versetzt, dass die Masse auch bei mehrtägigem Stehen beim Umkehren in der Flasche noch fliessend bleibt, aber weder dick noch gar fest wird. Die hierzu verwendete Salzsäure wird dem Volumen nach gemessen und alsdann das dreifache Volumen absoluten Alkohols nach und nach unter Umschütteln zugegeben. Hierbei findet starke Erwärmung statt und es löst sich hierbei viel $\mathrm{ZrCl}_{4}$ auf, welches sich aber beim Erkalten und dem nachfolgenden Verdünnen der Masse mit reinem Äther wieder fast vollständig abscheidet. Auch der Zusatz von reinem, trockenem Äther hat nach und nach unter Umschütteln zu erfolgen, bis zehn Volumtheile Äther (auf ein Volumtheil Salzsäure) zugegeben sind. Nach mehrstündigem Stehen filtrirt man das $\mathrm{ZrCl}_{4}$ ab, bringt es vom Filter herunter und rührt es mit einer Mischung von 1 Volumen conc. reiner 
Salzsäure, 3 Volumina absolutem Alkohol und 40 Volumtheile reinen Äthers an, worauf filtrirt, zunächst noch mit dieser Mischung, zuletzt aber mit reinem Äther gewaschen wird, bis dieser nicht mehr gelb, sondern farblos abläuft. Man kann das $\mathrm{ZrCl}_{4}$ durch Wiederbolnng dieser Operation absolnt eisenfrei erhalten, doch empfiehlt sich, wenn man mit grösseren Mengen arbeitet, eine Wiederholung nicht, und ist die weitere Reinigung des $\mathrm{ZrCl}_{4}$ in: anderer Weise vorzunehmen.

Nach dieser Behandlung wurde das natronhaltige Zirkonchlorid in Wasser gelöst, von etwas abgeschiedenem Chlorsilber (aus dem Silbertiegel) getrennt, mit etwas essigsaurem Natron versetzt und mit Sehwefelwasserstoff vollständig ausgefällt, die geringe Menge Niederschlag, bestehend aus $\mathrm{Cu}, \mathrm{Bi}$ und Sn entfernt, bis zum Weggehen des Schwefelwasserstoffs erhitzt, mit Salmiak versetzt und mit Ammon ausgefällt. Das bis zum Verschwinden der Chlorreaction gewaschene Zirkonerdehydrat wurde noch zweimal in Salzsäure gelöst, mit Ammon gefällt, gewaschen, zuletzt auf dem Wasserbade getrocknet, fein zerrieben und gesiebt. Ich will es hier als "rohes Zirkonerdehydrat" bezeichnen. Die bei diesen Operationen abgegangenen Waschwasser enthielten neben Natron, Kalk und Magnesia.

Das Gewicht des auf diese Weise aus $400 \mathrm{Grm}$. „rohen Zirkonerdenatron", das ist also aus circa $400 \mathrm{Grm}$. Zirkonkrystalle erhaltene, , o h eZirk o nerde hydrat"betrug $283.5 \mathrm{Grm}$. Es lieferte $77-78 \%$ Glührỉckstand, während reines $\mathrm{Zr}(\mathrm{OH})_{4}$ bei Umwandlung in $\mathrm{ZrO}_{2} \quad 77 \cdot 1$ Glührïickstand liefern sollte. Eine kleine Menge dieses "rohen Zirkonerdehydrates" in Salzsäure gelöst, noch zweimal mit Ammon gefällt, in Chlorid umgewandelt und wiederholt mit der erwähnten Mischung von Salzsäure, Alkohol und $\ddot{A}$ ther behandelt, lieferte vollkommen eisenfreies $\mathrm{ZrCl}_{4}$ als fein krystallinisches, schneeweisses, seidenglänzendes Pulver, das 50\% Glührückstand lieferte, während reines $\mathrm{ZrCl}_{4}$ $52.5 \%$ Glïhrücksstand an $\mathrm{ZrO}_{2}$ liefern sollte. Man ersieht hieraus, dass die erwähnte Mischung von Salzsäure, Alkobol und $\ddot{A}$ ther das Zirkonchlorid im Wesentlichen als neutrales und nicht als basisches Chlorid fällt.

Das "robe Zirkonerdehydrat" enthält noch kleine Mengen von: $\mathrm{Fe}, \mathrm{Zn}, \mathrm{Cu}, \mathrm{Mg}, \mathrm{Pb}, \mathrm{Ca}$ und $\mathrm{Na}$. Aber gerade wegen 
Verarbeitung und qualitative Zusammensetzung des Zirkons. 341

der Geringfügigkeit dieser Verunreinigungen ist es nicht leicht, dieselben zu beseitigen, da die Zirkonerde als amorphe Verbindung wie als Hydroxyd, basisches Sulfat, basisches Chlorid oder als basisches Acetat gefällt, stets einen Theil dieser Verunreinigungen mit sich niederreisst und auch eine Wiederholung der Operation nicht zum Ziele führt.

So ist die Fällung der Zirkonerde durch Kaliumsulfat in der Siedhitze als Mittel der Reinigung empfohlen worden, aber es gelingt auf diese Weise nicht einmal das Zirkonsulfat auch bei Wiederholung der Fällung eisenfrei zu erhalten.

Ich habe bei dieser Gelegenheit die nirgends erwähnte Beobachtung gemacht, dass der Niederschlag wohl in gesättigter Kaliumsulfatlösung fast vollkommen unlöslich ist, nicht aber in Wasser. Es löst sich bei fortgesetztem Waschen in dem Maße, als das Kaliumsulfat entfernt wird, eine beträchtliche Menge auf, während der Rest des Sulfates dann gänzlich unlöslich sich erweist. Es zeigte sich, dass der ursprüngliche Niederschlag. zwei verschieden geartete Zirkonsulfate einschloss, von welchen das eine wohl in Kaliumsulfatlösung, nicht aber in Wasser unlöslich, das andere aber in beiden Fällen unlöslich war. Das lösliche Salz sowohl wie das unlösliche, verhielten sich nämlich bei einer Wiederholung des Versuches ganz gleich, indem beide, wie die ursprïngliche Zirkonlösung von Neuem je ein lösliches und ein unlösliches basisches Sulfat lieferten. Die in beiden Salzen enthaltene Erde zeigte keinerlei Unterschied und bestand aus derselben Zirkonerde.

Erwähnen will ich bei dieser Gelegenheit der Zirkonacetate. Versetzt man eine neutrale Lösung von Zirkonchlorid mit hinreichend viel Natriumacetat und erwärmt die verdünnte Lösung. mehrere Stunden im Wasserbade, so fällt alles Zirkon als voluminöses, flockiges, basisches Zirkonacetat aus, aber keineswegs frei von den Verunreinigungen, obgleich die überstehende Lösung sehr stark sauer von freier Essigsäure ist.

Das basische Acetat löst sich vollständig in erwärmtem Eisessig (selbst in der Kälte gefälltes Hydrat nicht). Aus dieser Lösung wird das Zirkonacetat beim Verdunsten in Form einer spröden, rissigen, in jeder Beziehung dem arabischen Gummi vergleichbaren Masse erhalten. Beim Übergiessen mit Wasser 
quillt dieses Salz zunächst zu einer durchscheinenden Gallerte auf, bildet eine dicke, klebrige Masse, die dann zum Syrup und zu einer dicklichen, sehr leicht schäumenden Flüssigkeit wird. Der Versuch, die in Lösung vorhandenen krystallisirbaren Acetate durch Dialysiren zu entfernen, schlug fehl; selbst nach wochenlangem Verweilen auf dem Dialysator konnte nicht einmal Natrium entfernt werden.

Um ein reines Zirkonpräparat zu erhalten, versuchte ich auch den folgenden Weg. Das neutrale Chlorid wurde, nachdem es nochmals mit Schwefelwasserstoff behandelt worden, in einen grossen Überschuss einer gesättigten Lösung von kohlensaurem Ammon eingetragen und mit Schwefelammon ausgefällt. Der unter den nöthigen Vorsichtsmassregeln abgeschiedene Niederschlag enthielt zwar, wie das Funkenspectrum seiner Lösung ergab: $\mathrm{Fe}, \mathrm{Zn}, \mathrm{Pb}$, Ca und $\mathrm{Mg}$, allein in der Lösung war trotzdem neben Zirkonerde noch etwas $\mathrm{Fe}$, dann aber $\mathrm{Zn}, \mathrm{Pb}, \mathrm{Cu}$ nnd $\mathrm{Ca}$ sowie $\mathrm{Mg}$. Anch zweimaliges erneuertes Fällen mit Ammon als Hydroxyd und Waschen bis zum Verschwinden der Chlorreaction, schaffte $\mathrm{Zn}, \mathrm{Ca}$, und $\mathrm{Mg}$ nicht aus der Zirkonerde. Man erkennt hieraus, wie schwierig es ist, durch die gewöhnlichen Trennungsmethoden kleine Beimengungen von $\mathrm{Cu}, \mathrm{Pb}, \mathrm{Zn}, \mathrm{Fe}$, $\mathrm{Ca}$ und $\mathrm{Mg}$ von Zirkonerde zu trennen. Das betreffende Zirkonerdechlorid war mindestens dreimal mit Schwefelwasserstoff behandelt, einmal mit Schwefelammon in ammoniakalischer Carbonatlösung und mindestens fünfmal mit Ammon als Hydroxyd gefällt worden, ohne von obigen Verunreinigungen dadurch getrennt worden zu sein.

Zur völligen Reinigung des Zirkonchlorids führt nur ein meh rmaliges Umikrystallisiren desselben aus heisser, concentrirter, rauchender Salzsäure vom spec. Gewicht $1 \cdot 17$, in welcher das Zirkonchlorid bei gentigender Menge an Säure in der Wärme völlig löslich ist, in der Kälte aber sich sebr reichlich wieder abscheidet. Auf die Schwerlöslichkeit des $\mathrm{ZrCl}_{4}$ in concentrirter Salzsäure ist schon früher aufmerksam gemacht worden und das Waschen des Chlorides mit solcher Säure als Mittel zur Reinigung empfohlen worden (Chevreul, Herrmann), doch nur das Umkrystallisiren führt rasch und vollständig zum Ziel. Die Verluste hierbei sind nicht sehr gross. So lieferten beispielsweise $79 \mathrm{Grm}$. $\mathrm{ZrCl}_{4}$ nach dreimaligem Umkrystallisiren aus je $100 \mathrm{CC}$. heisser 
Verarbeitung und qualitative Zusammensetzung des Zirkons. 343

Salzsäure, Absaugen der Mutterlauge und jedesmaligem Nachwaschen mit je $50 \mathrm{CC}$. kalter Salzsäure $52 \mathrm{Grm}$. gereinigtes $\mathrm{ZrCl}_{4}$ Den grössten Theil des in der abfiltrirten Salzsåure enthaltenen $\mathrm{ZrCl}_{4}$ kann man noch durch Zusatz von Weingeist und $\ddot{\text { Atther }}$ in etwas weniger reinem Zustande abscheiden. Das ätheralkoholhaltige Filtrat eingedampft, gab einen Rückstand, dessen Funkenspectrum in concentrirter Lösung: $\mathrm{Cu}, \mathrm{Pb}, \mathrm{Zn}, \mathrm{Fe}, \mathrm{Mg}$ und $\mathrm{Ca}$ erkennen liess. Diese Metalle konnten nun auf gewöhnlichem Wege von einander geschieden werden, so dass ihre sehr reinen und brillanten Funkenspectren zur Ansicht kamen.

Das Funkenspectrum der wässerigen Lösung des auf diesem Wege gereinigten $\mathrm{ZrCl}_{4}$ ergab keine einzelnen Spectrallinien mehr, sondern ein anfangs schwaches, aber bald sehr stark anftretendes continuirliches Spectrum, welches von der sich bildenden und weissglihend werdenden Zirkonerde herrührt. Dieser Umstand berechtigt mich, anzunehmen, dass das $\mathrm{ZrCl}_{4}$ alle ihm noch beigemengt gewesene Verunreinigungen verloren, sonst würden die so hellen Linien derselben wenigstens anfangs sichtbar gewesen sein.

Obgleich die wässerige Lösung des Zirkonchlorides kein Linienspectrum gibt, so gibt doch das eingetrocknete Salz im Öffnungsfunken ein sehr linienreiches Spectrum. Auch hier ver ursacht weissgiühende Zirkonerde ein starkes, continuirliches Spectrum, doch wird es bei einem Spectralapparat mit stärker dispersirenden Prismen, welcher schon wegen der grossen Nähe der einzelnen Linien erforderlich ist, möglich sein, eine grosse Zahl von Linien zu messen, und zwar vom grünen Theil des Spectrums angefangen gegen das brechbarere Ende zu. Ich werde auf das Spectrum des reinen $\mathrm{ZrCl}_{4}$ im Öffnungsfunken später bei einem anderen Anlasse noch zurtickkommen.

Die, in der durch Flusssäure nicht weiter angreifbaren Grundmasse der Zirkonkrystalle neben Zirkonerde vorhandenen anderweitigen, basischen Oxyde waren in dem salzsauren, ätheralkoholhaltigen Auszuge enthalten. Da die Gesammtmenge dieser Chloride aus $400 \mathrm{Grm}$. Zirkonkrystalle stammte, so kam die Untersuchung dieser Chloride einer mit 400 Grm. Zirkonkrystallen vorgenommenen vollständigen qualitativen Analyse gleich und man kann sich nicht wundern, dass das Ergebniss 
dieser Analyse etwas vollständiger ausfiel, als eine mit den tiblichen Mengen vorgenommene qualitative Untersuchung ausgefallen wäre, dass diese Analyse ein besonderes Interesse bietet, da im Verlaufe derselben nicht weniger als noch 16 andere Metalle neben Zirkonium aufgefunden wurden.

Wurde in dem mit salzsäurehaltigem Ätheralkohol gewaschenen $\mathrm{ZrCl}_{4}$ durch Schwefelwasserstoff sehon $\mathrm{Cu}, \mathrm{Bi}, \mathrm{Sn}$ zur Fällung gebracht und später dann noch $\mathrm{Cu}, \mathrm{Pb}, \mathrm{Zn}, \mathrm{Fe}$ abgeschieden, so lieferte der obenerwähnte Anszug selbst, mitSchwefe]wasserstoff einen Niederschlag, der beträchtliche Mengen von Sn, kleinere von $\mathrm{Cn}$, Spuren von $\mathrm{Pb}, \mathrm{Ag}$ und $\mathrm{Pt}$ einschloss. Die beiden letzten Metalle stammen aus dem Platindraht, der zum Umrühren, und aus dem Silbertiegel, der zum Aufnehmen der Schmelze beim Aufschliessen des Zirkons diente. Der Schwefelammoniumniederschlag liess beim Lösen in Salzsäure $\mathrm{C}_{0}$ als Rückstand. In die kalische Lösung der Oxyde ging $\mathrm{Al}$, aber kein anderes Metall. Beim Behandeln des Eisenhydroxyds mit Salmiak und Ammon gingen in Lösung: $\mathrm{Co}, \mathrm{Zn}, \mathrm{Mn}, \mathrm{Mg}$ und $\mathrm{Ca}$. Mangan war nur in Spuren, Cobalt in kleiner Menge vorhanden, um aber das $\mathrm{Zn}, \mathrm{Mg}$ und $\mathrm{Ca}$ gänzlich rom $\mathrm{Fe}$ zu trennen, musste die Fällung mit Salmiak und Amon dreimal wiederholt werden. $\mathrm{Zn}, \mathrm{Mg}, \mathrm{Ca}$ konnten mit grösster Schärfe mittelst des Funkenspectrums der Chloridlösung nachgewiesen werden. Mangan war undeutlicher.

Die helle Farbe des von $\mathrm{Zn}, \mathrm{Mn}, \mathrm{Co}$, Ca und $\mathrm{Mg}$ befreiten Eisenhydroxydes dentete auf die Gegenwart von Erden. Das Eisen wurde mittelst kohlensaurem Ammon und Schwefelammon gefällt und von den Erden getrennt. Das Filtrat lieferte beim Einkochen die Erden, deren gelbe Farbe schon die Gegenwart des Urans verrieth. Zugleich war etwas Zirkonerde zugegen, welche vollständig als basisch-schwefelsaure Zirkonerde durch überschüssiges Kaliumsulfat in der Siedhitze ausgefällt werden konnte. Die relativ helle Farbe der aus den löslichen Sulfaten mittelst Ammon ausgefällten Hydroxyde wies auf die Gegenwart einer weiteren Erde neben Uranoxyd hin. Sie konnte, wenn auch nicht ganz vollständig von Uran mittelst kochender Salmiaklösung getrennt werden, in welcher Lösung sich Uranoxyd nur spurenweise, Zirkonerdehydrat gar nicht löst. Durch Wiederholung 
Verarbeitung und qualitative Zusammensetzung des Zirkons.

der Operation konnte die Erde ziemlich vollständig von Uran getrennt werden.

Die Erde ist "Erbinerde" mit Ausschluss von Didym.

Das Uran wurde erkannt an den charakteristischen Uranreactionen, welche alle zutrafen und an dem so bezeichnenden Absorptionsspectrum der Lösung des Hydroxyds in koblensaurem Ammon, Salzsäure und Salpetersäure.

Das Erbium wurde erkannt an dem Verhalten seines Hydroxyds gegen kochende Salmiaklösung, worin es sich leicht und vollständig löst, an seinem Verhalten gegen kochende Lösungen von essigsaurem Natron, wobei keinerlei Fällung entsteht, während Zirkonerde vollständig gefällt wird, der Löslichkeit seines Sulfates und den so charakteristischen Absorptionsspectren der Lösungen des Hydrates in kohlensaurem Ammon, Salzsäure und Salpetersäure.

Die Zeichnungen der Fig. $A$, zeigen in Nr. I das Absorptionsspectrum der noch mit Uran vermischten Erbinerde

Fig. A.

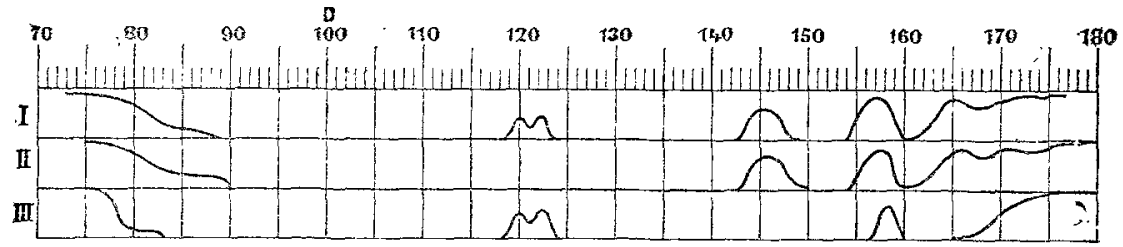

aus Zirkon, gelöst in kohlensaurem Ammon. Darunter Nr. II das Absorptionsspectrum einer Lösung von Uranoxyd, sowie weiter unten Nr. III einer Lösung von Erbiumoxyd, beide in kohlensaurem Ammon gelöst. Über das Vorhandensein von Uran und Erbium im Zirkon kann demnach kein Zweifel sein.

Da das Absorptionsspectrum der Chloride von Uran und Erbium aus Zirkon, Nr. 1, Fig. $\boldsymbol{B}$, bei 85 der Scale (beiläufige Wellenlänge 6590) im rothen Theile des Spectrums eine Absorptionslinie zeigt, welche weder dem Uranchlorid (Absorptionsspectrum Fig. $\boldsymbol{B}, \mathrm{Nr}$ II) noch dem Erbiumchlorid (Absorptionsspectrom Fig. $\boldsymbol{B}$, Nr. III) zukommt, so ist mit Rücksicht hierauf als auch mit Ritcksicht auf die Frage, ob denn ansser Erbium ron den Gadoliniterden im Zirkon keine anderen als Erbinm vorkommen, eine weitere Verfolgung dieses Gegenstandes wünschenswerth, was um so leichter sein wird, da ja die Abscheidung der 
Gadoliniterden aus Zirkon weit einfacher und auch vollständiger bewirkt werden kann, als dies hier in der vorstehenden Arbeit geschah, so lange man noch ganz im Unklaren sich befand, welcher Natur die vorhandenen Oxyde sein mochten. Die beobachtete Absorptionslinie $(\lambda=6590)$ gehört entweder einer der sehr seltenen Erden an, oder wahrscheinlicher, da diese Absorptionslinie keiner der bis jetzt beobachteten Absorptions-

Fig. B.

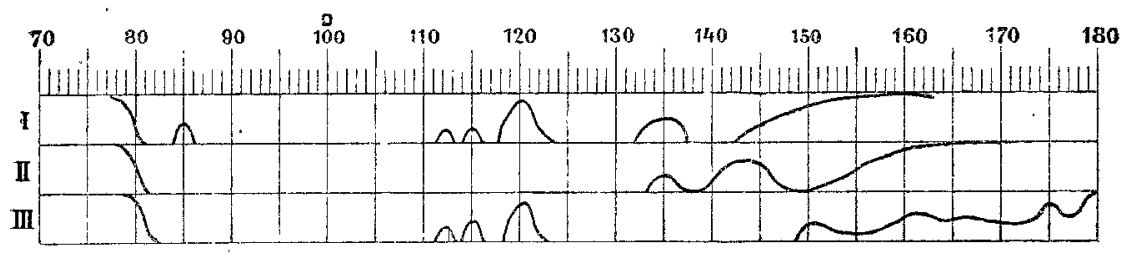

linien seltener Erden hinreichend nahe kommt, einem neuen Metalle. Zur Lösung dieser Frage gedenke ich entweder bedeutend grössere Mengen von Zirkon zu verarbeiten, oder solche Zirkone zu verwenden', welche wie Hyacinthen und Jargone durch ihre Absorptionslinien einen beträchtlicheren Gehalt an Uran und absorbirende Verbindungen verrathen.

Bekanntlich hat Sorby für gewisse Jargone eigenthümliche Absorptionsstreifen, und zwar im rothen, grünen und violetten Theile des Spectrums beobachtet. Er hielt diese Erscheinung, verursacht durch die Gegenwart eines neuen Elementes, welches er als "Jargonium" bezeichnete, fand aber später, dass diese Absorption dem Uran zuzuschreiben sei. Hierdurch erklärte sich wohl die Absorption im blanen und rothen Theile des Spectrums, nicht aber die beobachtete Absorption im mittleren grïnen Theile des Spectrums.

Diese Absorptionsstreifen rühren eben nach meinen eigenen vorliegenden Beobachtungen von Erbinerde her, und die Angabe Sorby's durch Zusammenschmelzen von reiner Zirkonerde und Uranoxyd mit Borax könne eine Perle erhalten werden, welche analoge Absorptionserscheinungen zeige, ist insoferne belanglos, als mir dieser Versuch mit meiner "reinen Zirkonerde" und Uran niemals gelang, offenbar, weil kein Erbium mehr in der Zirkonerde vorhanden war, die Absorptionen entsprachen nur der Gegenwart des Urans. 
Verarbeitung und qualitative Zusammensetzung des Zirkons. $\mathbf{3 4 7}$

Ich kehre nun zu dem weiteren Ergebnisse der qualitativen Analyse des salzsäurehaltigen Ätheralkoholauszuges des rohen Zirkonchlorides zurück. Aus dem Filtrate nach dem Schwefelammoniumniederschlage wurde durch Kochen zunächst noch etwas Co abgeschieden. Das Flammenspectrum ergab: $\mathrm{Ka}, \mathrm{Na}, \mathrm{Li}, \mathrm{Ca}$. Das Funkenspectrum der Lösung: Mg, Ca, Ka, $\mathrm{Na}$, Li.

In der krystallinischen Grundmasse des Zirkons sind demnach folgende metallische Grundstoffe enthalten:

$\mathrm{Sn}, \mathrm{Pb}, \mathrm{Cu}, \mathrm{Bi}, \mathrm{Zr}, \mathrm{Al}, \mathrm{Fe}, \mathrm{Co}, \mathrm{Mn}, \mathrm{Zn}, \mathrm{Mg}$, Ur, Er, Ca, Ka, Na, Li und man sieht hieraus, dass die Bezeichnung des Zirkons als ein „Polykrasilith" eine wohlberechtigte und zutreffende wäre. 